\title{
$\operatorname{con} f_{i} 131018--36$
}

PAPER

\section{COMPIITATIONAL MODEL OF SURFACE ABLATION FROM TOKAMAK DISRUPTIONS}

\author{
David Ehst and Ahmed Hassanein \\ Argonne National Laboratory \\ 9700 S. Cass Avenue \\ Argonne, IL 60439 USA
}

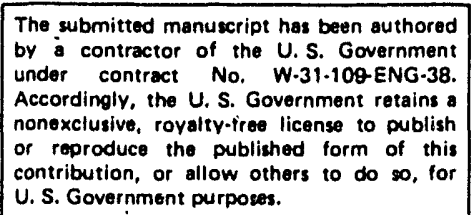

U. S. Government purposes.

October 1993

* Work supported by the U.S. Department of Energy, Office of Fusion Energy, under Contract No. W-31-109-Eng-38.

Paper to be presented at the IEEE/NPSS 15th Symposium on Fusion Engineering, October 11-15, 1993, Hyannis, MA. 


\title{
Computational Model of Surface Ablation from Tokamak Disruptions*
}

\author{
David Ehst and Ahmed Hassanein \\ Argonne National Laboratory \\ 9700 South Cass Avenue, Argonne. IL. 60439
}

\begin{abstract}
Energy transfer to material surfaces is dominated by photon radiation through low temperature plasma vapors if tokamak disruptions are due to low kinetic energy particles ( $<100 \mathrm{eV})$. Simple models of radiation transport are derived and incorporated into a fast-running computer routine to model this process. The results of simulations are in rair agreement with plasma gun erosion tests on several metal targets.
\end{abstract}

\section{INTRODUCTION AND SUMMARY}

Observed damage to tokamak surfaces during thermal disruptions has motivated bench tests (electron beams [ebeams] and plasma guns) which simulate tokamak conditions; and also models of plasma-material interactions have been produced in order to better understand the nature of this erosion process. Computer studies with codes such as A*THERMAL [1] have shown that the ablation cloud itself absorbs considerable energy from the thermal dump, and this vapor shielding serves to mitigate the actual damage to the solid surface. Vapor shielding of e-beams is easily calculated from the stopping power of high-energy electrons in the vapor. We have recently developed a computer routine DESIRE, which extends this analysis to sub-keV particles, more typical of thermal disruptions and plasma guns. Such low-energy particles suffer $90^{\circ}$ scattering and create a thermal plasma source in front of the vaporized target material. In this limit, energy is radiated as photons by the source plasma; this radiation is partly absorbed in the target vapor and partly transmitted through the target to the materiai surface. Under typical conditions $\left(10 \mathrm{MJ} / \mathrm{m}^{2},<1 \mathrm{~ms}\right.$ pulse width) the source impulse maintains both the source and target plasmas in the Saha regime. For given densities and vapor temperatures the code computes the degree of ionization and the effective charge state of both zones. The heat capacity of each vapor, which can be a significant heat sink in this process, is found from particle kinetic energies along with the ionization and excitation energy. The source and target plasmas respectively radiate and absorb radiation over a broad spectrum of wavelengths; and the optical depth of each zone is computed at each wavelength by calculating the contributions from Bremsstrahlung (with ions and neutra!s) and free-bound radiation. Finally, the equation of radiative transfer determines the photon power spectrum transmitted through the target vapor to the material surface.

Considerable energy is lost out the sides of the source vapor zone; as this radiated power is directed away from the ablating surface, this two-dimensional effect further serves to reduce ablation.

* Work supported by the USDOE/OFE, under contract No. W-31-109-Eng-38.
Our computer code simultaneously solves these equations in a time-dependent manner, conserving energy and following the evolution of the target vapor cloud. The code uses simplified algorithms which eliminate many detailed atomic physics calculations which have little bearing on the final result. As a consequence, we have achieved a very fastrunning routine which makes parametric studies practical for disruption modelling. Details of our atomic physics calculation compare well with more sophisticated computer codes, arid the ablation predictions substantially agree with measurements of plasma gun erosion of targets such as $\mathrm{Fe}$ and Mo. Results reported in this paper are based on a stand-alone version of DESIRE, while more accurate simulations are possible by incorporating DESIRE as a subroutine to A*THER.MAL.

\section{EXAMPLE: HYDROGEN GUN ABLATION OF Mo}

Our computational model was tested by comparison with observable parameters on several plasma gun experiments. As an illustration we model an experiment with the PLADIS gun [2] at the University of New Mexico. We consider a hydrogen gun with proton and electron energies of $30 \mathrm{eV}$ and $1 \mathrm{eV}$, respectively, incident on a Mo surface, and we study the case with $U_{1}=9.1 \mathrm{MJ} / \mathrm{m}^{2}$ heat load over a $100 \mu$ s duration.

The gun hydrogen plasma radiation source and the vaporized Mo target plasma are both followed in a time dependent manner.

Fig. 1 shows the evolution of the source and target vapor temperatures, $\theta_{\mathrm{S}}$ and $\theta_{\mathrm{T}}$, and ionization degree, $\Phi_{\mathrm{S}}$ and $\boldsymbol{\Phi}_{\mathrm{T}}$. The target vapor is initially only barely ionized, as $\theta_{\mathrm{T}}=0.45$ $\mathrm{eV}$ is well below the first ionization potential of Mo $\left(\chi_{1}=\right.$ $7.1 \mathrm{eV}$ ), but $\Phi_{\mathrm{T}}$ increases rapidly as $\theta_{\mathrm{T}}$ rises due to the heating by the source vapor's radiation. The source vapor starts out and remains fully ionized until $t \cong 10 \mu \mathrm{s}$. During this first phase the hydrogen radiates inefficiently, but as $\theta_{\mathrm{S}}$ eventually drops to $\sim 6 \mathrm{eV}$ the recombination radiation absorption edges arise, leading to a spike in the hydrogen radiated power. As a consequence of this power loss the source temperature abruptly collapses. Beyond $\mathrm{t} \cong 30 \mu \mathrm{s}$ the hydrogen radiative loss diminishes; and both source and target vapors are only partially ionized, with slowly dropping temperatures.

Fig. 2 shows the spectral emissivity of the source plasma and transmissivity of the target vapor at $\mathrm{t}=13 \mu \mathrm{s}$. The Lyman and Balmer absorption edges are prominent for the hydrogen source at $\sim 6 \mathrm{eV}$ (during the radiative collapse). The effect of this free-bound radiative opacity is the two peaks in the source emissivity, $e_{s}$, near $\eta_{s}=h c / K \theta_{S} \lambda=0.6$ and 2.3 in Fig. 2. Note, however, $e_{s}(\eta)$ is quite small compared to black body 
emissivity. The target vapor at $1 \mathrm{eV}$ exhibits the absorption edge of $\mathrm{Mo}^{+}$recombination into the Mo ground state; the corresponding wavelength of $0.17 \mu \mathrm{m}$ is at $\eta_{\mathrm{s}}=1.1$ for a source temperature $\theta_{S}=6.1 \mathrm{eV}$. Due to the small lineaveraged density of vaporized Mo, the target vapor is optically thin, with the target transmissivity nearly equal to the source emissivity.

Despite the transparency of the target vapor, relatively little of the plasma gun energy arrives at the Mo surface; the fraction is only $Y=0.45 \mathrm{MJ} / 9.1 \mathrm{MJ}=0.049$. Most of the incident energy is radiated by the source vapor sideways $(6.8$ $\mathrm{MJ} / 9.1 \mathrm{MJ})$ and backward $(0.55 \mathrm{MJ} / 9.1 \mathrm{MJ})$. At the end of the pulse the source vapor also stores considerable energy density due to its heat capacity $\left(\mathrm{U}_{\mathrm{S}} / \mathrm{U}_{1}=1.2 \mathrm{MJ} / 9.1 \mathrm{MJ}\right)$. The target vapor stores relatively little energy density and losses little to sideways radiation. Due to these sundry heat sinks the vapor shielding is quite effective, the ablation depth being only $\delta=0.29 \mu \mathrm{m}$ for Mo with $p=9000 \mathrm{~kg} / \mathrm{m}^{3}$.

\section{DESIRE CODE COMPARISON WITH PLASMA GUN DATA}

Code validation is best achieved by comparison with experiments, which we have done for various metal surfaces and different plasma gun conditions. The calculations assume a sine-shaped wave form for the heat load, of total length 100 $\mu \mathrm{s}$.

Fig. 3, adapted from [2], shows three data points of measured energy transmission fraction to a tungsten calorimeter through the vapor clouds generated under specified heat loads. The gun energy density, noted by the abscissa, was determined from discharges into a deep copper bucket calorimeter, which accounted for total plasma energy, including sideways-radiated losses. When the DESIRE code is run with a fixed initial ion kinetic energy from the plasma gun, $\mathrm{E}_{\mathrm{io}}=30 \mathrm{eV}$, the calculation shows $\mathrm{Y}$ decreasing as $\mathrm{U}_{1}$ increases from $6 \mathrm{MJ} / \mathrm{m}^{2}$ to $16 \mathrm{MJ} / \mathrm{m}^{2}$. Yet the data suggest $Y$ increases with $U_{l}$; so we conclude that $E_{j o}$ may be increasing with $U_{1}$. (This is not unreasonable, as, for a fixed pulse width, e.g., $100 \mu \mathrm{s}$, the experimental set-up keeps the bank capacitance constant and increases $U_{l}$ by raising the voltage on the bank.) Whereas $E_{i o} \cong 30 \mathrm{eV}$ agrees with the data at $U_{1}$ $=6 \mathrm{MJ} / \mathrm{m}^{2}$, apparently $\mathrm{E}_{\mathrm{io}}>100 \mathrm{eV}$ would be needed to duplicate the data at $16 \mathrm{MJ} / \mathrm{m}^{2}$.

Target erosion is an obvious test for DESIRE. The experimental data (points) in Fig. 4 [2] display the average ablation depth of steel found at four different values of $U_{1}$. There is a wide, inconsistent scatter of results with only slight differences in target composition, so we cannot expect DESIRE to fully explain the observed erosion. However, it is evident, in the experiment and from the DESIRE results for pure $\mathrm{Fe}$, that minimal erosion occurs below $\mathrm{U}_{1} \equiv 5 \mathrm{MJ} / \mathrm{m}^{2}$. Yet substantial erosion occurs for $\mathrm{U}_{1}>10 \mathrm{MJ} / \mathrm{m}^{2}$. The code results show this onset of erosion is associated with an ablation threshold, which is a characteristic of the thermal properties of metal surfaces. Note also that the DESIRE curve at $\mathrm{E}_{\mathrm{io}}=30 \mathrm{eV}$ is in good agreement with the data at $\mathrm{U}_{1} \approx 12$ $\mathrm{MJ} / \mathrm{m}^{2}$, while it underestimates erosion at $16 \mathrm{MJ} / \mathrm{m}^{2}$. This is additional evidence suggesting the plasma gun produces higher ion kinetic energies at large $U_{1}$.

The agreement of the DESIRE model with the experiment, shown in Fig. 4 , is much better than the MAGFIRE result [3] which found $\delta=10.7 \mu \mathrm{m}$ at $\mathrm{U}_{1}=8.3$ $\mathrm{MJ} / \mathrm{m}^{2}$. This emphasizes the importance of the additional heat sinks incorporated in our treatment, which constitutes a distinct improvement relative to earlier calculations.

Molybdenum targets were tested with both U.S. and Russian plasma guns, and the experimental data, in Fig. 5, again show $[4,5]$ how typically there is a scatter of results under similar conditions. The horizontal error bars from VIKA are a measure of uncertainty in the energy content delivered in the plasma pulse, and the vertical scatter of PLADIS points indicate a certain lack of reproducibility in these experiments. Some difference between the two institutional results may be atuibuted to subtle geometric differences; for example, VIKA had a gun-to-target distance of $3.0 \mathrm{~m}$ but PLADIS had gun electrodes much closer to the target. The DESIRE results shown treated both geometries the same. Our calculated erosion with $\mathrm{E}_{\mathrm{io}} \cong 30 \mathrm{eV}-50 \mathrm{eV}$ is in approximate agreement with the data. Time-of-flight measurements on PLADIS suggest the leading edge of the gun pulse is populated by protons at a few tens of electron volts, which is perhaps a bit lower than $E_{\text {io }} \cong 30 \mathrm{eV}-75 \mathrm{eV}$ reported on VIKA.

Erosion of tungsten was likewise measured on both VIKA and PLADIS, and the results are shown in Fig. 6 along with the DESIRE calculations. In the experiments tungsten appears more robust than Mo under the same heat pulse conditions.

Both experiment [4] and the DESIRE theoretical model show beryllium to suffer more erosion than the three metals discussed above, which is evident from Fig. 7. A noteworthy point illustrated in the graph is that the central dcpth of the damage crater is roughly triple the average depth, as inferred from mass loss measurements. The nonuniform crater depth may be associated with a depression in the melt layer, possible redeposition or splashing of the melt toward the edges, and nonuniform heat deposition by the gun plasma. Such details are not included in the present DESIRE model.

\section{CONCLUSIONS}

Although our atomic physics models are very simplified we are able to duplicate many observed features of plasma gun ablation experiments with a reasonable accuracy. Besides energy transmission fraction and erosion depth, our model agrees well with measured vapor temperatures, pressures, and spatial extent as seen in experiments [4-6].

The main shortcoming of our model presently is its limitation to a pure hydrogen plasma source with $\mathrm{E}_{\mathrm{io}}+\mathrm{E}_{\mathrm{eo}} \tilde{<}$ $50 \mathrm{eV}$. We hope that by extending the code capability to 

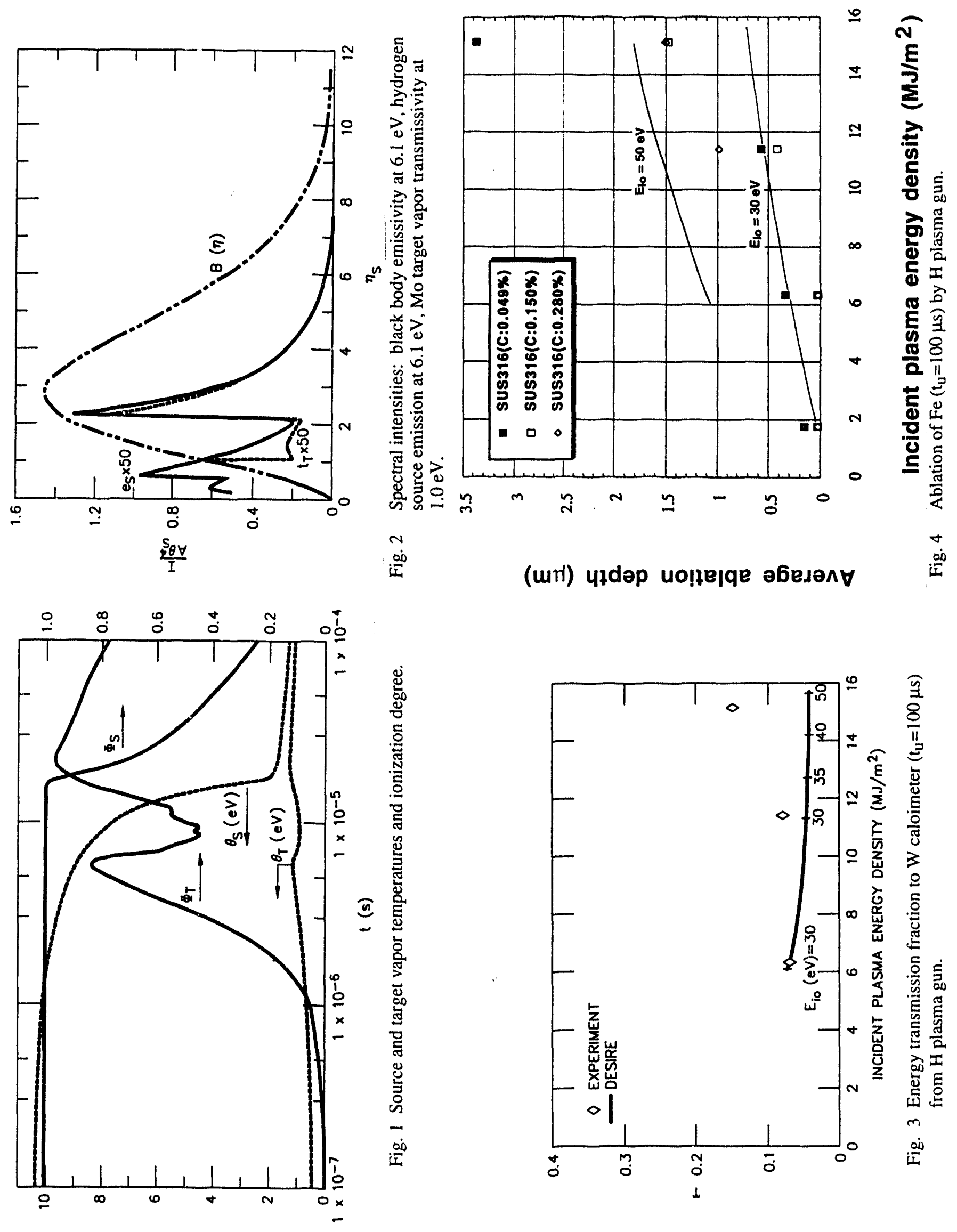

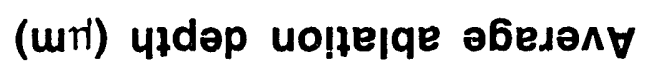

送

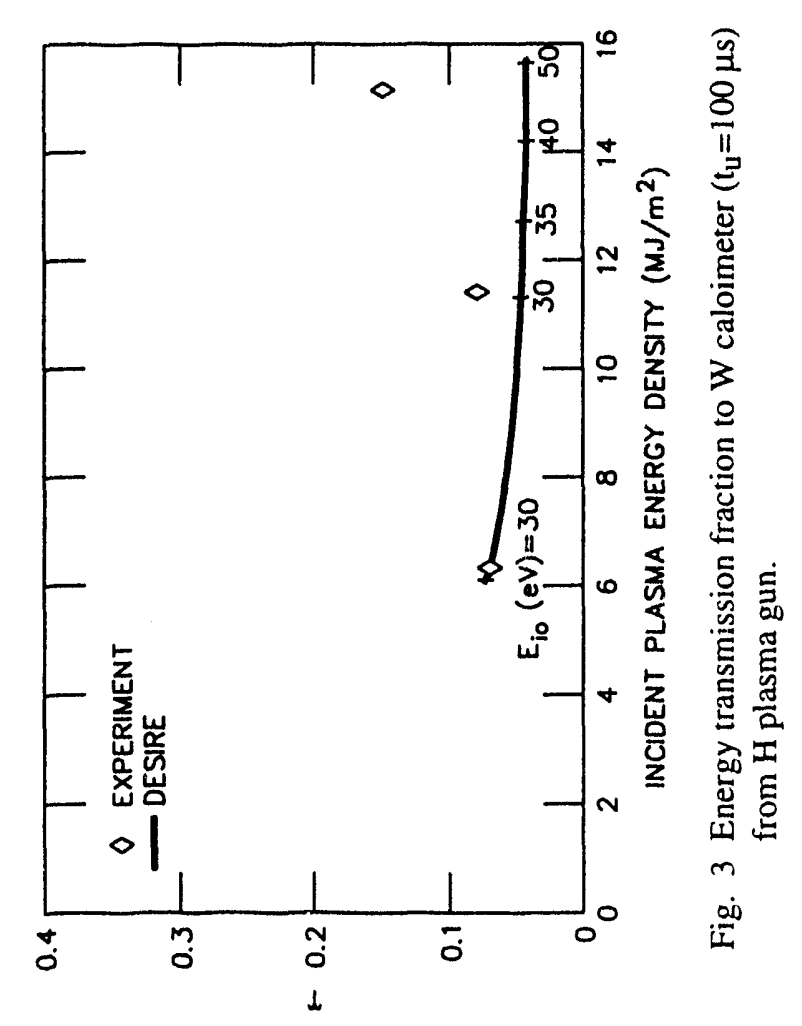


energies $>100 \mathrm{eV}$ we will be better able to duplicate the experimental data, such as shown in Figs. 3 and 7.

\section{ACKNOWLEDGMENTS}

We are indebted to R.R. Peterson for many enlightening conversations, and to J.M. Gahl, J.T. Bradley, III, and J.F. Crawford for access to their plasma gun data.

\section{REFERENCES}

[1] A. M. Hassanein, "Simulation of plasma disruption induced melting and vaporization by ion or electron beam," J. Nucl. Mater. 122\& 123 (1984) 1453.

[2] S. Suzuki, J. F. Crawford, J. T. Bradley, III, and J. M. Gahl, "Experimental study of pulse plasma heat flux absorption and ablation measurements of materials during the simulation of the thermal quench phase of tokamak plasma disruption," J. Nucl. Mater. 200 (1993) 265.

[3] J. Gilligan, D. Hahn, and R. Mohanti, "Vapor shielding of surfaces subjected to high heat fluxes during a plasma disruption," J. Nucl. Mater. 162-164 (1989) 957.

[4] J. M. Gahl, private communication.

[5] V. R. Barabash et al., "Damage of refractory metals and carbon-based materials under simulation of the thermal influence at plasma disruption," Fusion Eng. and Design 18 (1991) 145.

[6] V.M. Strunnikov, private communication.

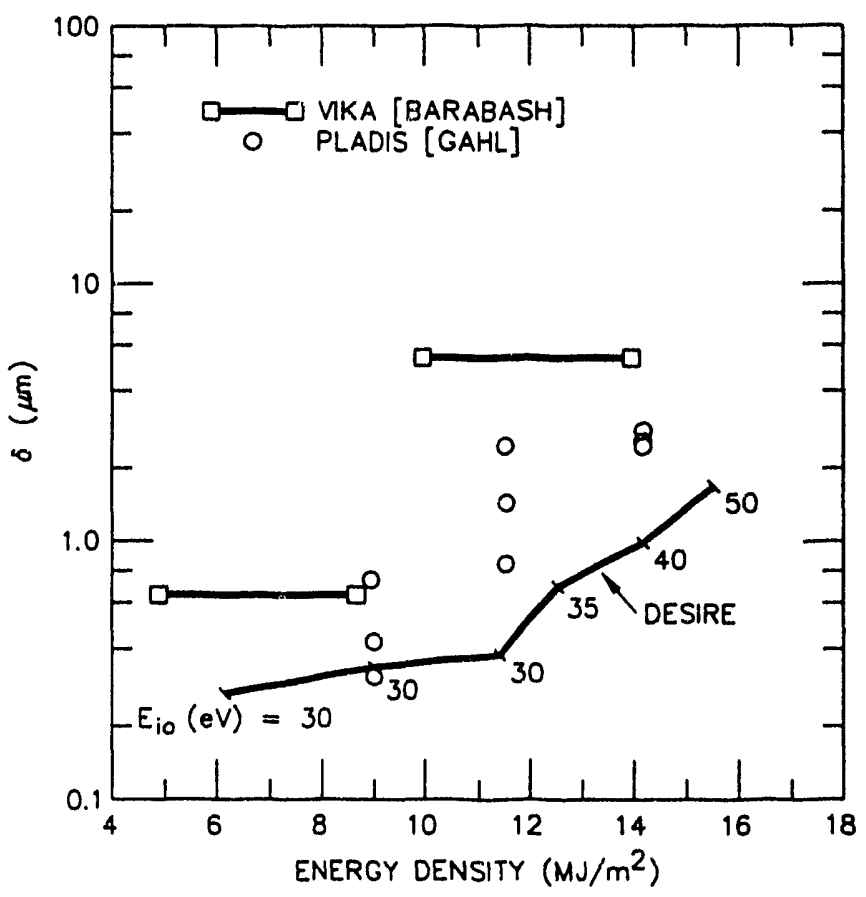

Fig. 5 Ablation of Mo $\left(t_{u}=100 \mu\right.$ s) by $\mathrm{H}$ plasma guns.

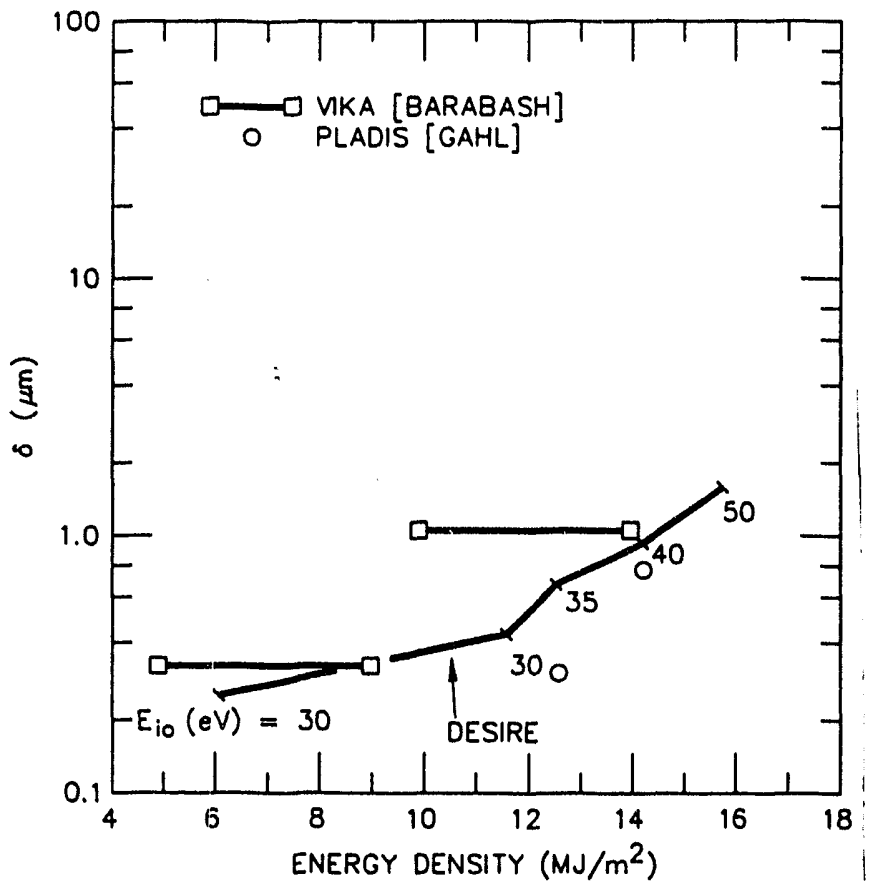

Fig. 6 Ablation of $\mathrm{W}(100 \mu \mathrm{s})$ by $\mathrm{H}$ plasma guns.

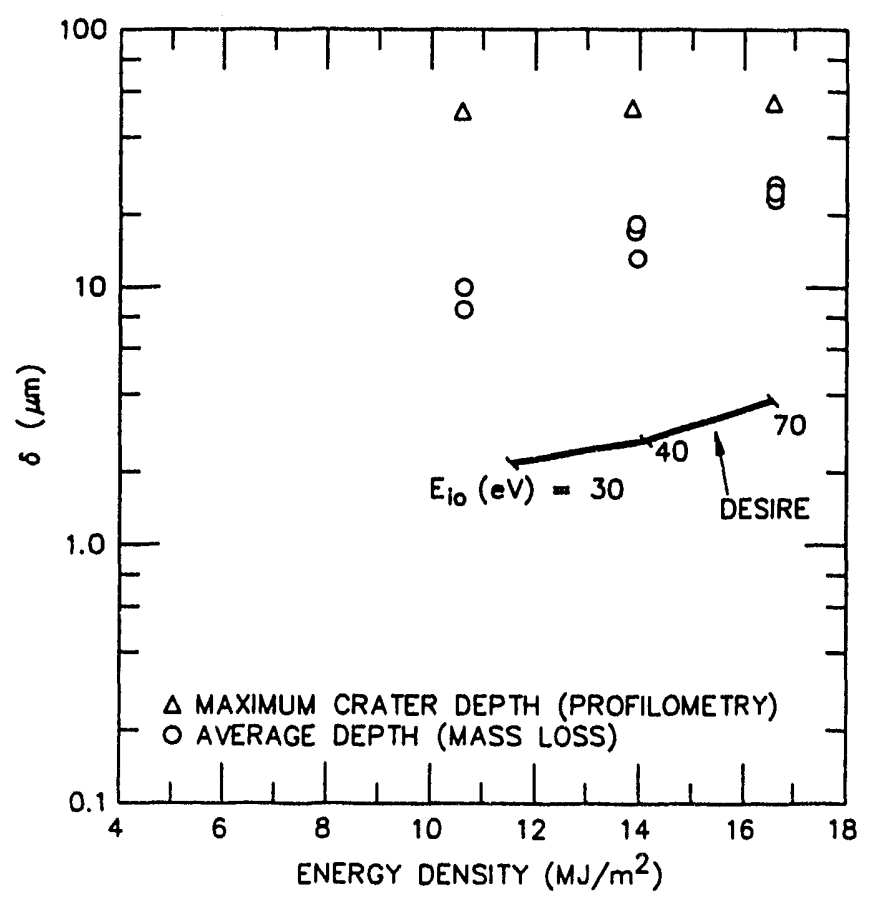

Fig. 7 Ablation of $\mathrm{Be}(100 \mu \mathrm{s})$ by $\mathrm{H}$ plasma gun.

\section{DISCLAIMER}

This report was prepared as an account of work sponsored by an agency of the United States Government. Neither the United States Government nor any agency thereof, nor any of their employees, makes any warranty, express or implied, or assumes any legal liability or responsibility for the accuracy, completeness, or usefulness of any information, apparatus, product, or process disclosed, or represents that its use would not infringe privately owned rights. Reference herein to any specific commercial product, process, or service by trade name, trademark, manufacturer, or otherwise does not necessarily constitute or imply its endorsement, recommendation, or favoring by the United States Government or any agency thereof. The views and opinions of authors expressed herein do not necessarily state or reflect those of the United States Government or any agency thereof. 
